\title{
A Non-Destructive Impedance Method Using Resonance to Evaluate the Concentration of Steel Fibers in Concrete
}

\author{
T. Bachorec ${ }^{1}$, P. Fiala ${ }^{2}$, M. Steinbauer ${ }^{1}$, Z. Roubal ${ }^{1}$ \\ ${ }^{I}$ Department of Theoretical and Experimental Electrical Engineering, FEEC, BUT, Technická 3082/12, 61600 Brno, \\ steinbau@feec.vutbr.cz,roubalz@feec.vutbr.cz,bachorec@feec.vutbr.cz \\ ${ }^{2}$ SIX Centre, FEEC, BUT, Technická 3082/12, 616 00, Brno, FEEC, BUT, Technická 3082/12, 61600 Brno; Czech \\ Republic,fialap@feec.vutbr.cz
}

\begin{abstract}
Steel-fiber reinforced concrete is a composite material characterized by outstanding tensile properties and resistance to cracking. The concrete, however, exhibits such characteristics only on the condition that the steel fibers in the final, hardened composite are distributed evenly. Current methods to evaluate the distribution and concentration in a fiber composite are either destructive or exhibit a limited capability of evaluating the concentration and orientation of the fibers. In this context, the paper discusses auxiliary techniques and laboratory tests that evaluate the density and orientation of the fibers in a composite material, presenting an innovative approach to impedance-based non-destructive testing. The actual methodology utilizes the resonance of the measuring device and the measured sample set; the desired state occurs within the interval of $f=10 \mathrm{kHz}$ and $2 \mathrm{GHz}$.
\end{abstract}

Keywords: Concrete, electric impedance, electromagnetic field, non-destructive testing, resonance, steel fiber.

\section{INTRODUCTION}

A substantial amount of present-day materials for technical and technological purposes, rather than being produced or modified solely from natural sources, comprise many individual components to form composite products. This group then also includes certain building materials, such as concrete, which embodies an elementary structural material consisting of reinforcement, bonding agents, and water. Within the preparation process, admixtures are added to the composite to ensure perfection in some parameters of its physical and mechanical properties. Importantly, the last decades have witnessed major development of fiberreinforced concrete, a material formed through the addition of a particular type of metal reinforcement, but also a general rise of interest in composite materials and related NDT methods [1]-[7], [8], [9].

In order for a material to exhibit the required mechanical and thermal properties, it is necessary to secure the relevant macroscopic properties, such as the homogeneous and isotropic distribution of the components, and thus also the resulting parameters. This phase depends on the selection of a suitable technology for preparing and manufacturing the given material. During the manufacturing process, however, the homogeneity (the concentration of the components of the composite) and isotropy (the orientation of the components) are verified and evaluated; such operations can be performed using various methods.
The initial laboratory-based techniques are destructive [5] and inconvenient for the verification of already functioning units. Intensive attention is thus paid to methods and principles that enable us to evaluate non-destructively the concentration of the components of a composite, reinforcement in particular; by extension, these approaches are also employed to assess the orientation of individual elements in a concrete reinforcing material [6], [10]-[12]. From the physical point of view, the propagation/dispersion of an electromagnetic (EMG) wave [12], [13] can be advantageously utilized from the lowest frequency bands to the highest ones, namely, the radiofrequency [14], infrared (heat propagation) [13], and X-ray or gamma radiation [9] ranges. The discussed set of procedures then facilitates designing novel methods [1], sensors, and devices to measure the above-outlined properties of composites.

An interesting tool consists of techniques and devices to evaluate the concentration of fibrous particles, or steel fibers, in both fresh and solidified concrete; in this case, however, the orientation of the fibers is assessable only with major difficulty.

Our experiments have shown that, utilizing the electric and ferromagnetic properties of steel fibers as a composite component in reinforced concrete, it is possible to monitor the concentration and distribution of the fibers in a nondestructive manner [1]. 


\section{IMPEDANCE-BASED EVALUATION OF COMPOSITE MATERIAL QUALITY VIA NDT}

The non-destructive methods presented to date, such as that discussed in papers [8], [14], [9], exploit the properties of the electromagnetic (EMG) field to monitor the distribution of fibers in the tested composite. To evaluate the attributes of the components of a composite, the actual principle employs the electrical impedance $\hat{Z}$ in the region of the monitored sample. The relevant formula then reads

$$
\hat{Z}=\frac{|\hat{\boldsymbol{E}}|}{|\hat{\boldsymbol{H}}|}
$$

where $\hat{Z}$ denotes the complex impedance of the harmonic behavior of the electric and magnetic field components, $\hat{E}$ is the complex vector of the electric field intensity, and $\hat{\boldsymbol{H}}$ denotes the complex vector of the magnetic field intensity. The details of the fields and other aspects are shown in, for example, Fig. 1.

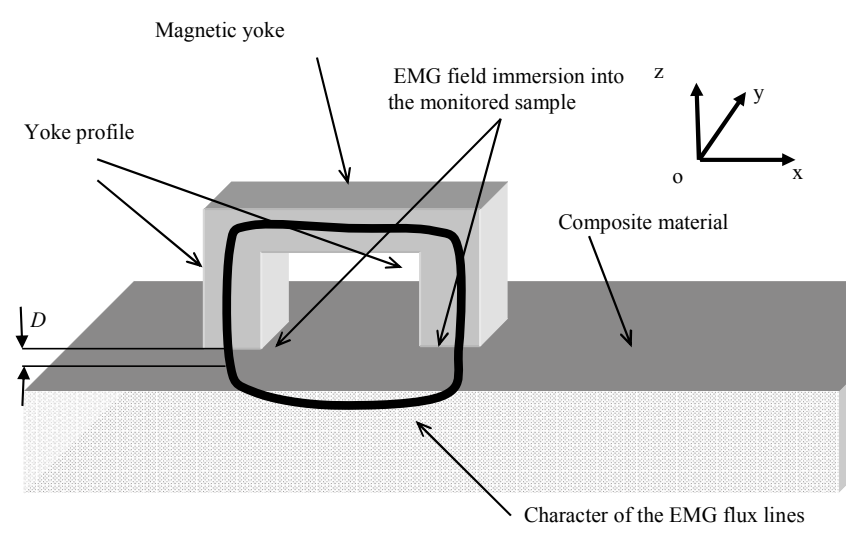

Fig.1. The principle of the impedance-based NDT evaluation of composite material quality.

An improved, more accurate methodology [1], [16], [18][19] was designed to expand the set of current impedancebased techniques. The novel approach is capable of evaluating the orientation and local densities of components of a composite, namely, components of a reinforcement containing electrically or electrically and magnetically conductive fibers. The proposed methodology to measure and evaluate the properties of a tested sample exploits effects of the electromagnetic field at conditions near the resonant state.

\section{APPLIED METHODOLOGY}

The procedure uses two effects: the electrical impedance of the employed medium permeated by an EMG wave (describable by such means as the lines of force of the magnetic field), Fig.1., and the resonance of the electromagnetic circuit of the coupled sensing element. Given the relevant geometry setting (dimensions A, B, C, D) and materials applied in the designed sensing device, whose basic constituent is a ferromagnetic yoke positioned at a suitable distance $D$ from the surface of the tested composite sample, resonances will occur at the selected frequency $f$ of the harmonic signal generator that supplies the excitation coil's winding (Fig.2.).

\section{A. Design of the measuring device}

The apparatus for the sensing and evaluation of the electromagnetic properties of ferromagnetic, electrically conductive parts of a composite material can be fabricated using a ferromagnetic yoke with an electrical winding; such a yoke is $\mathrm{C}, \mathrm{U}$, or E-shaped and has either a partially distributed or a fully uniform electrical winding, Fig.2.

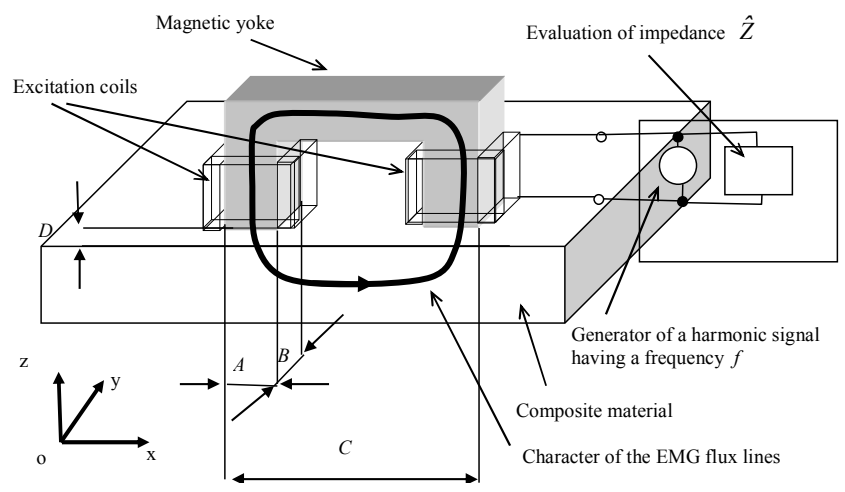

Fig.2. The basic configuration of the novel, NDT-based impedance sensor.

The winding is split between the yoke's arms to provide for strong magnetic coupling with the tested composite material, Fig.2. The detection device for the NDT is connected to a magnetic circuit designed in such a manner that its resonant frequency in a free space lies within the interval of $f=10 \mathrm{kHz}$ and $2 \mathrm{GHz}$ (Fig.2.) to facilitate series resonance in the sense of an equivalent diagram with concentrated parameters.

The appropriate selection of a frequency $f$ then already depends on the parameters of the tested composite material (including the mass density, volume $V$, or distribution of the ferromagnetic and electrically conductive components); the desired measurement depth from the surface of the composite material sample; distance $D$; and dimensions $A, B, C$ of the sensor. The detection and measuring device consists of a generator to excite magnetic flux $\Phi$ and a meter of electrical impedance $\hat{Z}$. The given ferromagnetic yoke is attached to the sample, with its electrical winding excited by a generator of voltage $u(t)$; the excitation coil carries the instantaneous value of current $i(t)$ [9], [16].

The impedance meter embedded in the external electric circuit, Fig.2., evaluates the complex impedance and its changes in both the component and the exponential forms. The frequency $f$ of the excitation circuit of the detection and measuring device (impedance meter, Fig.3.b) is set to be located at the heel of the resonance curve, Fig.3.a)), within the interval of $f_{\mathrm{d}}$ and $f_{\mathrm{r}}$.

In the designed device, the resonance effect will occur if the parameters are preset according to the equivalent model scheme, Fig.4., where the concentrated parameters are described by the inductance $L$, capacitances $C$, resistances $R$, 
and mutual inductance $M$. With the parameters set in this manner, the excitation frequency $f$ of the electric voltage generator $u(\mathrm{t})$ (of the external electric circuit) lies within the frequency interval of $f_{\min }=10 \mathrm{kHz}$ and $f_{\max }=2.0 \mathrm{GHz}$. For the selected material of the tested sample and the present distance $D$ of the magnetic yoke from the plane of the sample, the excitation frequency is set to the lower frequency $f_{\mathrm{d}}$ with the lower limit of the quality factor $Q_{\mathrm{d}}$ and to the upper frequency $f_{\mathrm{h}}$ with the upper limit of the quality factor $Q_{\mathrm{h}}$. The homogeneity of the examined volume $V$ can be described via the scheme of the equivalent model exhibiting concentrated parameters, which include the capacitance $C_{0}$, resistance $R_{0}$, and inductance $L_{0}$, as indicated in Fig. 4 .

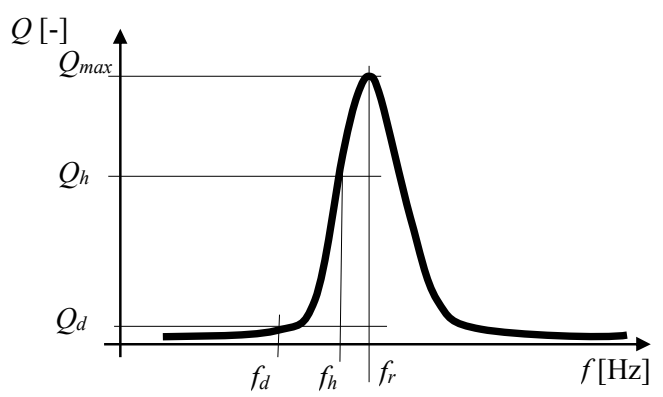

a)

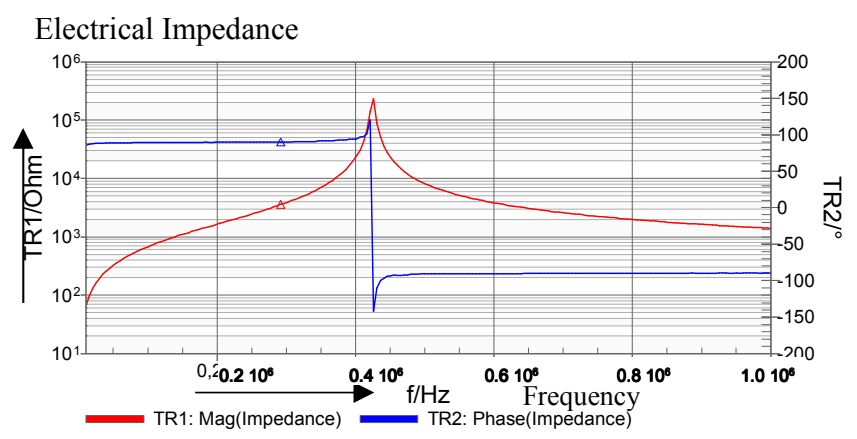

b)

Fig.3. The resonant conditions: a) the quality factor curve; b) the measured impedance module and phase of the magnetic yoke.

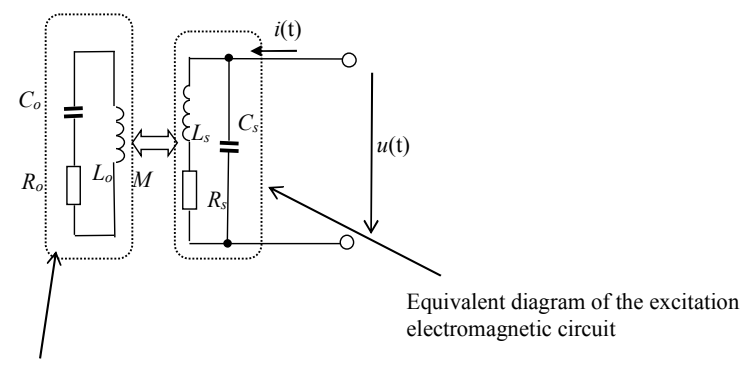

Equivalent diagram of the response of the EMG field of the monitored composite material sample

Fig.4. The electrical scheme of the NDT method for the resonant state.

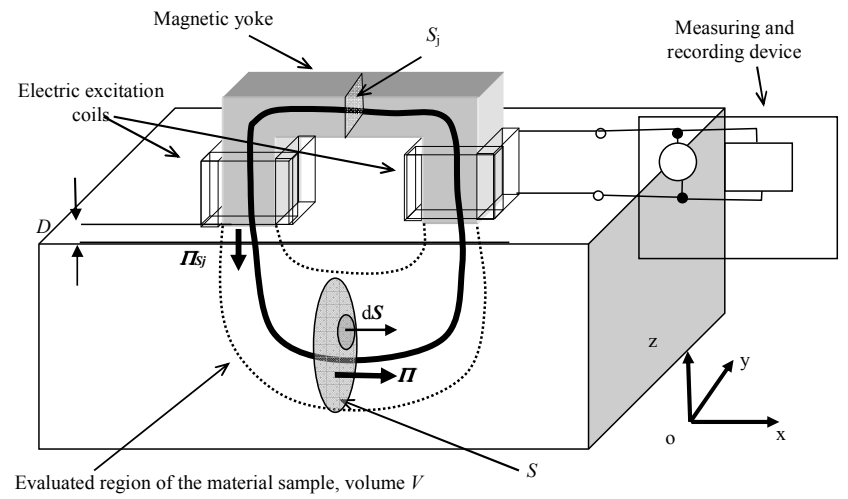

Fig.5. A scheme of the NDT resonance method: the evaluation of critical parameters.

The previously used procedure [17] for evaluating the homogeneity of composite material components via an assessment of the impedance $\hat{Z}$ differs from the technique discussed herein (namely, an approach to operate within an area close to series resonance, [1]) in that the impedance $\hat{Z}$ in the complex form and within the area close to series resonance is very sensitive to changes in the distribution of the monitored composite material component in the region of the sensed magnetic field [15], [17]. The sensitivity may be two to three orders of magnitude higher than that exhibited by currently used methods and methodologies. The evaluation and analysis of the complex impedance $\hat{Z}$ then enable us to assess, at a high sensitivity rate, the density of the composite material components and the orientation of the needle-like formations of the composite components.

The frequency $f$ of the impedance meter's excitation signal (Fig.2.) is progressively set such that the resonance quality factor $Q_{\mathrm{h}}$ could assume the values

$$
Q_{0.5}=\frac{1}{2} Q_{\max }, Q_{s q 2}=\frac{1}{\sqrt{2}} Q_{\max } \text { or } Q_{s q 3}=\frac{1}{\sqrt{3}} Q_{\max }
$$

without acquiring values lower than the magnitude of the factor $Q_{\mathrm{d}}$. In the experimental setting, the values proved to be beneficial for the final evaluation of the location with nonuniform distribution of the composite material components. To evaluate the mass density of the monitored composite material component, the electric power specific density vector is assessed; this vector is then written as

$$
\hat{\Pi}=\hat{E} \times \hat{\boldsymbol{H}},
$$

where the symbol $\times$ denotes the vector product. The dissipated electrical power $P$ generated in the monitored composite material component is bound to the surface density of the active power $\widehat{\Pi}$ from the above expression (3), and this dissipated power is formulated within

$$
P=\int_{S_{j}}\left|\hat{\Pi}_{S_{j}}\right| \cdot d \boldsymbol{S}
$$


where $P$ is the dissipated electrical power in the region having the volume $V$ in the measured portion of the composite material, $\widehat{\Pi}_{S_{j}}$ denotes the power flux surface density (Poynting vector) in the area of the shaping yoke at the distance $D$ from the surface of the composite material, $\mathrm{d} \boldsymbol{S}$ is the vector of the element of the cross section area of the measured part of the composite material sample, $S_{\mathrm{j}}$ represents the section through the magnetic yoke, and $S$ is the cross section of the area of the monitored material sample (Fig.5.).

For the measuring and recording device with a magnetic yoke, presented in Fig.2. and Fig.5., the complex impedance $\hat{Z}$ in the exponential form contains components of the electric and magnetic fields (having the intensities $E$ and $H$ ) of the given configuration of the magnetic circuit and the composite material sample, Fig.4. The complex impedance form is written as

$$
\hat{Z}_{0}=\frac{\|\hat{\boldsymbol{E}}\|}{\|\hat{\boldsymbol{H}}\|} \angle \varphi_{0},
$$

where $\angle \varphi_{0}$ denotes the phase of the complex number in the exponential form, $\|\hat{\boldsymbol{E}}\|$ is the module of the electric field intensity vector, and $\|\hat{\boldsymbol{H}}\|$ is the module of the magnetic field intensity vector. The values of the impedance $\hat{Z}$ from the above formula (5) are obtainable from the measuring and recording device, or impedance meter, and can be written as follows:

$$
\hat{Z}_{0}=\frac{|\hat{u}|}{|\hat{i}|} \angle \varphi_{0},
$$

where $|\hat{u}|$ is the measured value of the module of the instantaneous value of electric voltage, $|\hat{i}|$ denotes the measured value of the module of the instantaneous value of electric current, $\widehat{U}$ represents the complex form of the electric voltage on the terminals of the electric coil with equivalent expression via the concentrated parameters from Fig.4., and $\hat{I}$ is the complex form of the electric current flowing through the electric coil. For the preset resonant frequency $f_{\mathrm{r}}$ of the entire setup comprising the described detection and measuring device and the electric coil wound on the arms of the ferromagnetic yoke, there holds - for the complex impedance $\hat{Z}$ in the exponential form - the formula

$$
\left.\hat{Z}_{0}\right|_{f_{r}}=\frac{\|\hat{\boldsymbol{E}}\|}{\|\hat{\boldsymbol{H}}\|} \angle \varphi_{0}, \varphi_{0} \cong 0 .
$$

For the component form, the complex impedance is written as

$$
\left.\hat{Z}_{0}\right|_{f_{r}}=Z_{0, \boldsymbol{R e}}+j Z_{0, \boldsymbol{I m}}, \forall f=f_{r} \text { holds } Z_{0, \boldsymbol{I m}} \cong 0,
$$

where $Z_{0, \text { Re }}, Z_{0, \operatorname{Im}}$ are the real and imaginary components of the complex impedance $\hat{Z}$. Based on such measured data, it is then easy to evaluate the magnitude of the dissipated power (4), which corresponds to the mass density of the monitored composite material component

$$
P=Z_{0, \boldsymbol{R e}} I^{2}
$$

where $I$ is the module of electric currents closing in the monitored area of the tested composite material sample. If the entire resonant system is not set within the area of resonance, as indicated in Fig.2. and Fig.5., the complex impedance $\hat{Z}$ changes, and we have

$$
\left.\hat{Z}_{0}\right|_{f \neq f_{r}}=\frac{\|\hat{\boldsymbol{E}}\|}{\|\hat{\boldsymbol{H}}\|} \angle \varphi_{0}, \varphi_{0} \neq 0 .
$$

The change of resonance for the initial preset frequency $f_{\mathrm{r}}$ of the detection and measuring device and the connected electric coil will occur if the position of the ferromagnetic yoke is altered such that, in the monitored volume $V$ of the tested composite material sample, we can observe a variation of the mass density or orientation of the needle-like formations of the monitored composite material components.

Using the above-expressed relationships between the mass density of the composite material component and the electromagnetic field, (3) to (9), it is possible to evaluate the parameters of the desired properties of the composite material samples; these parameters include, for example, the density of the reinforcement formations, density changes of the monitored component in axes $x, y$ of the system of coordinates (Fig.5.), orientation of the elementary formations of the composite components, and change of the monitored component's orientation in axes $x, y$ of the system of coordinates.

If the frequency $f$ of the measuring device is set to $f_{0.5}$, namely, such that the resonance corresponds to $Q_{0.5}=$ $\frac{1}{2} Q_{\max }$, very sensitive setting can be utilized for the detection of changes in the mass density of the composite material components in coordinates $x, y$ of the system of coordinates, according to the above formulas (3) to (9). Setting the frequency $f$ of the measuring device to $f_{\mathrm{sq} 2}$ in such a manner that the resonance corresponds to $Q_{s q 2}=\frac{1}{\sqrt{2}} Q_{\max }$ then results in less sensitive sensing of the non-uniform volume distribution of the composite material components in the variable coordinates $x, y$ of the system of coordinates (Fig.5.); however, this procedure is also suitable for evaluating the magnitude of the mass density of the monitored component in the composite material sample via the dissipated power $P$, according to formula (9). Further, the frequency $f$ of the impedance meter can be set to $f_{\mathrm{sq} 3}$, namely, such that the resonance corresponds to $Q_{s q 3}=\frac{1}{\sqrt{3}} Q_{\max }$; this approach enables us to evaluate the mass density of the monitored component and, simultaneously, is sufficiently accurate to recognize changes in the orientation of the monitored component of the needle-like composite material in the variable coordinates $x, y$ of the system of coordinates. 


\section{B. Methodology to evaluate the monitored composite material parameters}

Commonly used NDT-based impedance measurement techniques and methods to evaluate materials both at their surfaces and in the immediate vicinity [11]-[12], [14], [16][17] exploit various configurations of the magnetic yoke and electric coils, including the arrangement shown in Fig.5. The authors of the present paper nevertheless propose that there still remain ample perspectives for further development of a methodology to utilize the indicated configuration of the magnetic circuit and the measured sample of the tested material in establishing a procedure capable of highsensitivity detection and evaluation of the monitored parameters of the composite material components. Within our research, a methodology [1], [16] to detect variation in the monitored parameters at a substantially increased degree of sensitivity was identified. This approach and its individual phases can be characterized as follows:

- In the first step, the electric excitation coils located on the arms of a C, U, or E-shaped ferromagnetic yoke (Fig.2.) are supplied by the measuring device (Fig.5.) with electric voltage $u(\mathrm{t})$ to create an instantaneous value of electric current $i(\mathrm{t})$, which, at a frequency $f$, excites in the magnetic yoke a magnetic field exhibiting a magnetic flux $\Phi$. The frequency is set to a value $f_{\text {sq3 }}$ such that there occurs resonance characterized by the quality factor $Q_{s q 3}=\frac{1}{\sqrt{3}} Q_{\max }$ from Fig.3. The magnetic yoke is positioned at a pre-selected, and constant for the following steps, distance $D$ from the surface of the monitored composite material sample. Using the measuring and recording device (Fig.5.), the complex impedance $\hat{Z}$ is then recorded in both the component and the exponential forms.

- Subsequently, within the second step, the position of the magnetic yoke is changed via rotating it along the axis of one of the magnetic yoke's arms, and setting according to the first step is performed. The rotation angle $\alpha$ (Fig.6.) corresponds to, for example, $10^{\circ}$; then, we measure and record the complex impedance $\widehat{Z}$ in both the component and the exponential forms.

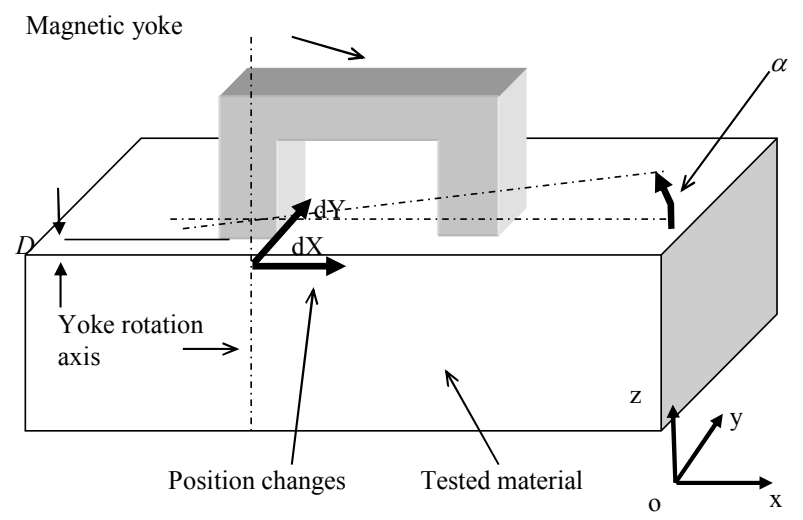

Fig.6. The magnetic yoke position changes during the evaluation of critical parameters.
- The third stage consists of changing and recording the complex impedance $\widehat{Z}$ in accordance with the second stage, and we apply anew the rotation angle to change the position of the magnetic yoke until the arm has rotated by $360^{\circ}$.

- In the related fourth step, the results from the first to the third steps are employed to evaluate, using the above formulas (3) - (9), the impedance $\widehat{Z}$ and the dissipated power $P$. According to the expressions (3) and (4), we can specify at the tested location the mass density ratios of the monitored components, namely, the composite reinforcement consisting of ferromagnetic or ferromagnetic and electrically conductive formations of the composite material sample. It is then possible to determine the dissipated power $P_{100}$ obtained for the tested volume $V$ (Fig.7.) with the material of the element component reinforced at $100 \%$, and this dissipated power can be compared with the measured dissipated power $P$. Using the related quotient, the proportional representation of the monitored composite material component in the observed volume $V$ is acquired.

- Within the fifth stage, the frequency $f$ of the detection and measuring device is set to $f_{0.5}$ in such a manner that the resonance and quality factor correspond to the value of $Q_{0.5}=\frac{1}{2} Q_{\max }$ for the original point of measurement and the distance $D$. After that, measurement is performed in accordance with the second and third steps of the methodology, and, exploiting the data thus obtained, we evaluate the distribution homogeneity and orientation of the formations of the monitored component of the composite material sample at the original point of measurement; the output is graphically interpretable.

- The sixth step lies in that the excitation values of the electric coil are set to the frequency $f_{\text {sq2 }}$, exciting an electromagnetic field having a frequency $f$ for resonance with the quality factor of $Q_{s q 2}=\frac{1}{\sqrt{2}} Q_{\max }$, and the position of the magnetic yoke (Fig.6.) changes such that the yoke is shifted from the original point of measurement by a distance $\mathrm{d} X$ and a distance $\mathrm{d} Y$. These distances, $\mathrm{d} X$ and $\mathrm{d} Y$, are oriented with respect to the surface of the monitored composite material sample, and the defined distance $D$ of the magnetic yoke front from the surface of the monitored composite material sample. In the newly set position, the complex impedance $\widehat{Z}$ in both the component and the exponential forms is measured and recorded.

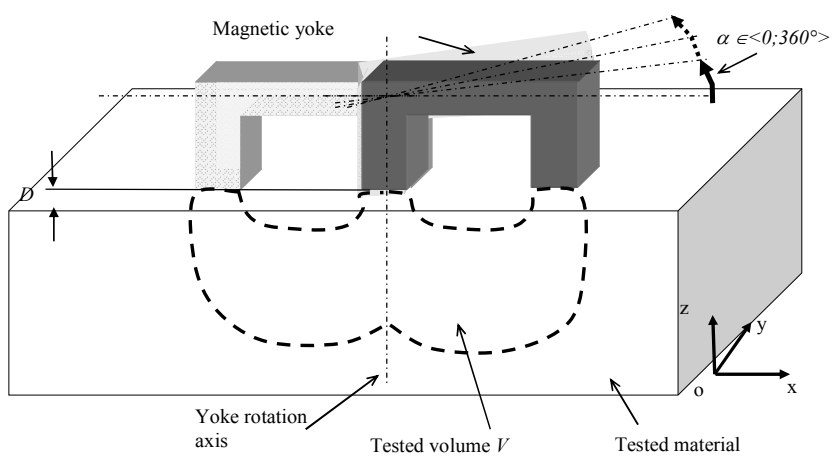

Fig.7. The evaluation of critical parameters in the volume $V$. 
Then a shift by the distances $-\mathrm{d} X, \mathrm{~d} Y ; \mathrm{d} X,-\mathrm{d} Y$ and $-\mathrm{d} X,-\mathrm{d} Y$ with respect to the original point of measurement (based on the first step of the methodology) is performed, and the complex impedance $\widehat{Z}$ in both the component and the exponential forms is measured and recorded. Such measurements facilitate a more precise evaluation of the density in the volume $V$ of the monitored component in the tested composite material sample.

- The related seventh stage comprises positioning the ferromagnetic core into a new measurement point; this new position should be, in the direction of the coordinate $x$, different by at least a distance greater than the length $C$ of the basis plus double the arm width $A$ equaling $C+2 A$, according to the dimensions (Fig.2.) of the magnetic yoke. After setting the discussed position of the magnetic yoke, we measure, record, and evaluate the quantities in accordance with the first to the sixth steps of the presented methodology, thus obtaining the numerical and graphical evaluation of the distribution, density, and orientation of the monitored component of the tested composite material sample.

\section{APPLIED METHODOLOGY}

The methodology tests as described above were performed in laboratory conditions. The actual testing of the composite material sample (with the needle-like reinforcement components) for the selected type and version of the magnetic yoke within the given frequency range is shown in Fig.8. The material sample had been fabricated to support the testing of the proposed NDT methodology for the evaluation of magnetic impedance, and it enabled us to verify and compare not only the anticipated properties of the applied impedance method and instrumentation but also the analyses achieved with the discussed methodology. We monitored and evaluated the limits of attainable evaluation accuracies to identify possible effects that contribute towards type A and B uncertainties.

A series of measurements were performed to test, in a suitable magnetic yoke, the shape and dimensions, the related parameters of distance $D$, and the frequency $f$; all these factors were examined to facilitate the resonance setting while the measurement uncertainties are minimized. Several magnetic yokes were designed experimentally, with tuning to $f=10 \mathrm{kHz}-10 \mathrm{MHz}, \quad 50-100 \mathrm{kHz}, \quad 10 \mathrm{kHz}-40 \mathrm{MHz}$ (Fig.8.), and $10 \mathrm{kHz}-450 \mathrm{kHz}$. Considering the abovespecified yokes and frequency ranges, the setting suitable for the NDT and the applied detection system was found to lie within $f=10 \mathrm{kHz}-2 \mathrm{MHz}$, depending on the concrete composite material sample tested, its actual composition, and representation of the monitored components of the composite.

An exemplary impedance evaluation related to selected frequencies is shown in Fig.9.a), Fig.9.b). The behavior of the impedance module $Z$ indicates that the representation and distribution of the composite material components strongly affects the shape of the frequency characteristics. The designed methodology was employed to evaluate the impedance $\hat{Z}$ in both the component and the exponential forms for one point of measurement (Fig.8.).
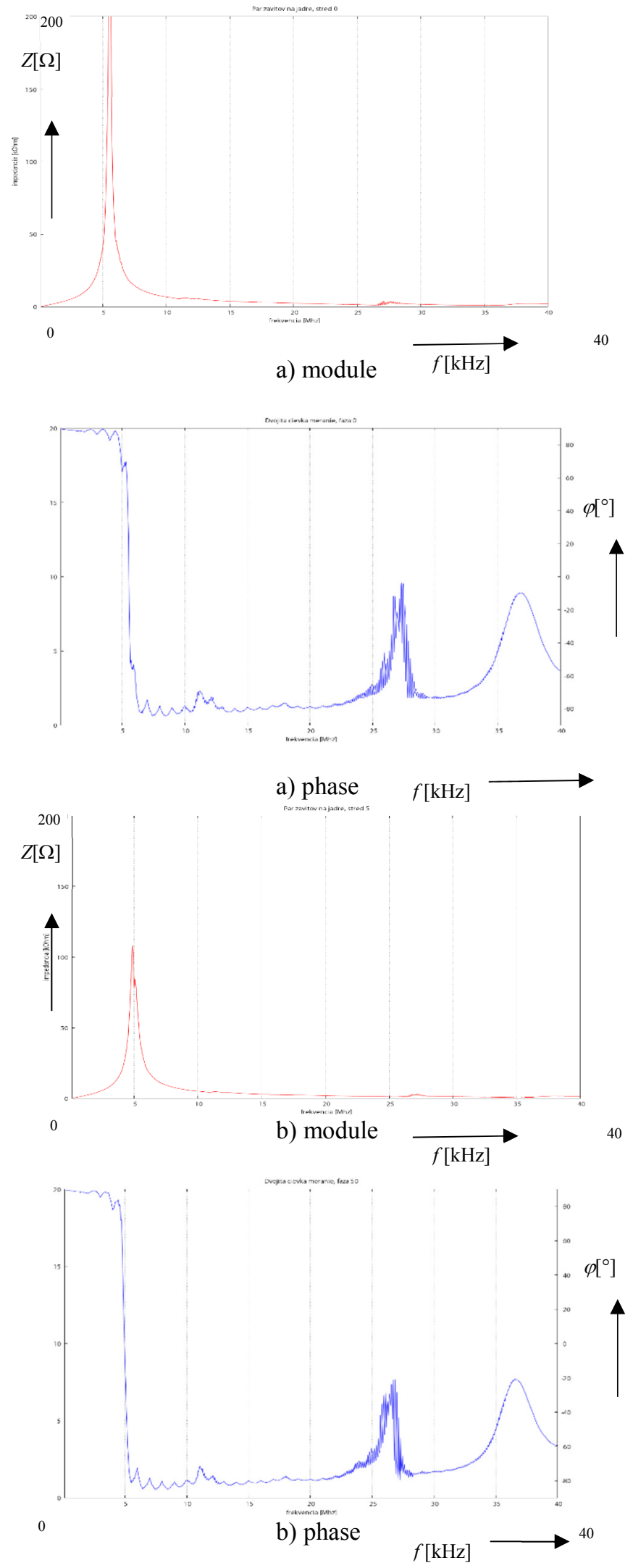

Fig.8. The test sensing of the impedance $\widehat{Z}$ for the selected frequency range of $f=0-40 \mathrm{MHz}, f_{\mathrm{r}}=4.95 \mathrm{MHz}$ to evaluate the properties of the composite material sample depending on the rotation angle of the magnetic yoke axis $\alpha$. a) The impedance for axis $\alpha=0^{\circ}$; b) the impedance for axis $\alpha=40^{\circ}$. 


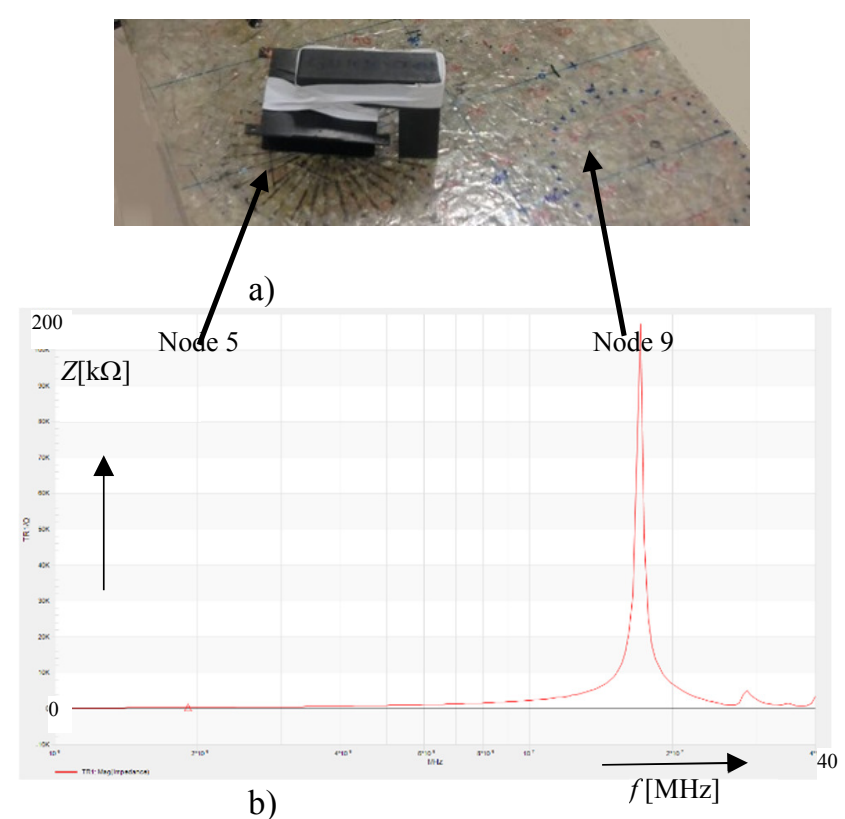

Fig.9. a) Testing the examined composite material sample, $f=16.5 \mathrm{MHz}$; b) the laboratory-based measurement to evaluate the module of impedance $Z$ in the resonance region: the relationship between the impedance and the frequency, $f=16.5 \mathrm{MHz}$.

The laboratory test to evaluate changes (Fig.11. - Fig.14.) of the orientation distribution homogeneity in the needle-like formations of the composite material reinforcement followed the above-characterized methodology and is presented in Fig.10., which contains color marks located at the given points of measurement to graphically illustrate the main orientation directions of the needle-like formations in relation to the reinforcement density. The prevailing orientation of the formations is defined by the direction of the marks, and the relevant density follows from their sizes: a longer mark denotes a higher value, whereas a shorter one expresses a lower rate. The relevant densities are directly proportional to the magnitudes of the power dissipation presented in the above formulas (4) - (10), enabling us to determine the direct volume of the monitored component in the sample; alternatively, the comparative method can be used, in which the density value is compared with the phantom-based measurement, and the interval of the content of the composite component is defined. Fig.10. compares two composite material samples; values are recorded for measurement points 5 and 9 on the tested sample.

The semi-automatic manipulation and impedance recording device is displayed in Fig.14.; structurally, the device comprises a ferrite magnetic yoke, Fig.12.b), and basic measuring blocks, Fig.13. These components are configured and installed such that the applied remotely controlled drive will position the system (Fig.14.b)) above the point to be measured, and the subsequent procedures are as follows: the data recording mode is selected; the magnetic yoke is rotated (Fig.7.b)) to reach the desired positions, consistently with the seven-stage methodology outlined above; the relevant measurement data are recorded (Fig.14.c)); and the manipulation program (Fig.14.d)) moves the system to another location, as described within the corresponding operating stage. The output data format is shown in Fig. 15. The given data can then be further processed using standard IT tools, via table or graphics processors.

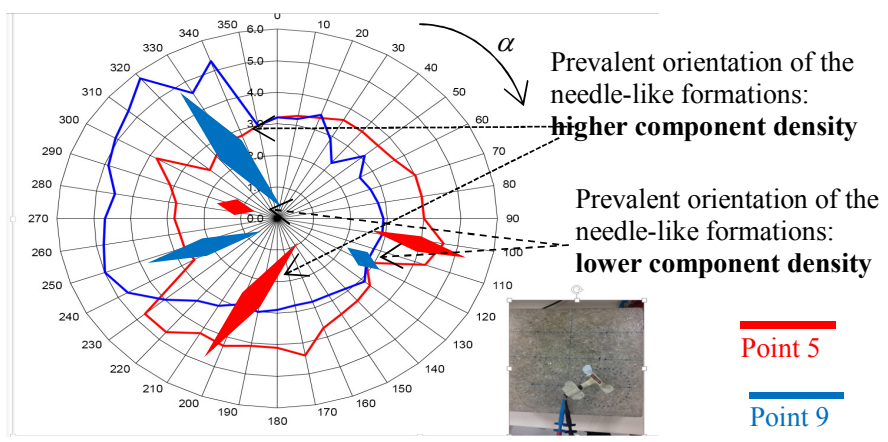

a)

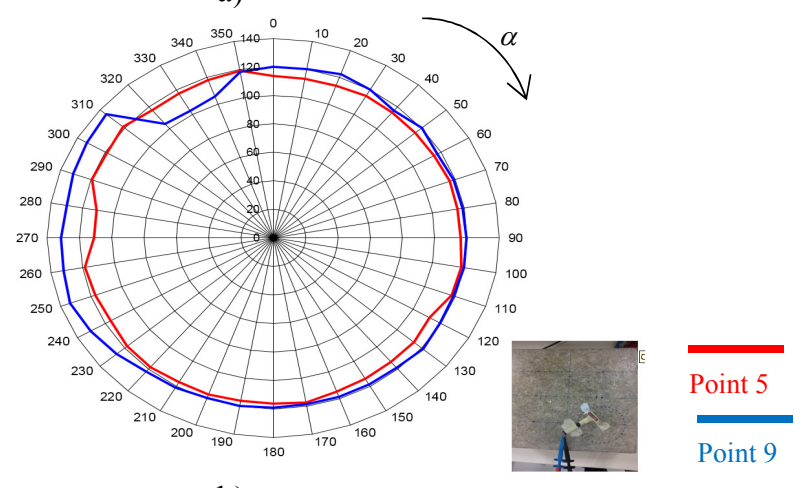

b)

Fig.10. The laboratory-based evaluation of the impedance $\widehat{Z}[\Omega$, deg]: a) the module, b) the phase. Both of these components are related to measurement points 5 and 9 on the test sample, $f=16.5 \mathrm{MHz}$.

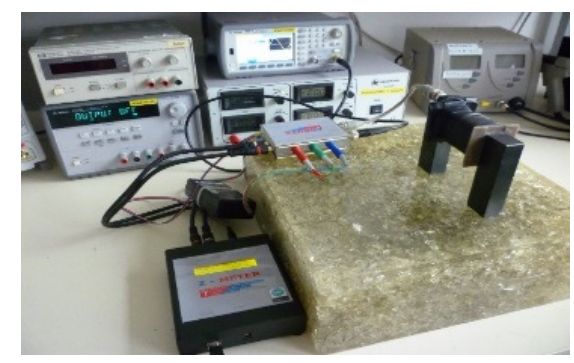

Fig.11. The equipment for the laboratory-based evaluation of relevant factors the basic module comprising the analog and digital components of the impedance/phase meter; the magnetic yoke; and the tested sample.
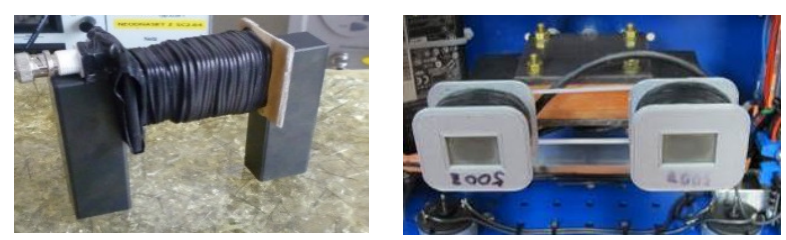

Fig.12. The single-purpose measurement and recording device (NDT) to evaluate the impedance module/phase and the concentration/orientation of the monitored component of the composite material sample. 


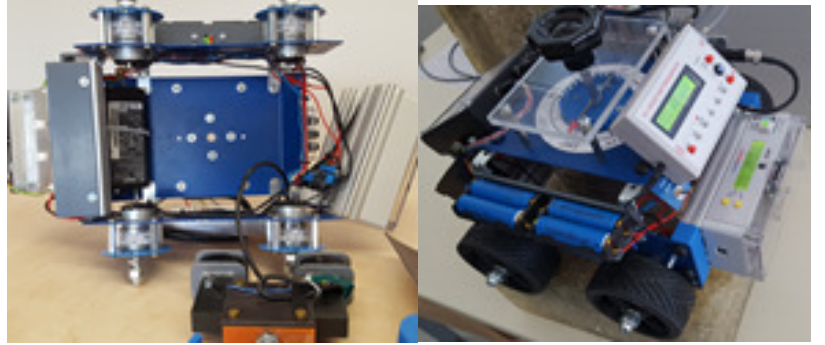

a)

b)

Fig.13. The compact NDT measurement and recording device to evaluate the impedance module/phase of the monitored component of the composite material sample: a) the basic functional blocks of the manipulator; b) the complete semi-automatic monitoring system;

\section{CONCLUSION}

A novel methodology using impedance in the complex form to facilitate NDT-based measurement and evaluation of composite material components was designed, described, and tested; the approach substantially increases the sensitivity and accuracy in the evaluation of the monitored component. Test samples of composite materials with needle-like formations applied as the reinforcement were experimentally measured under laboratory conditions; in this context, the authors derived and proved the basic functional principles of the NDT methodology for evaluating the distribution, density, and orientation of ferromagnetic conductive fibers in a composite material. This novel metrological approach towards the NDT of composite materials exploits impedance analysis.

The single-purpose devices designed and tested to support the NDT methodology facilitated a substantial sensitivity improvement, allowing more precise evaluation of the monitored components.

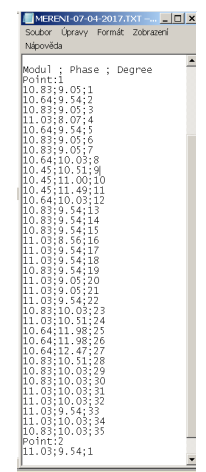

a)

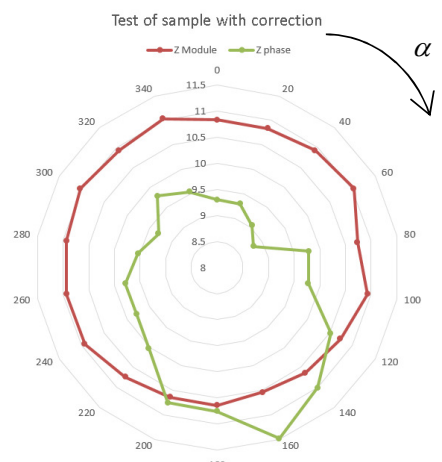

b)
Fig.14. The tested sample: a) the stored data format; b) the related interpretation.

\section{ACKNOWLEDGEMENT}

The research was financed by the National Sustainability Program under grant No. LO1401 and supported within a grant of the Czech Science Foundation (GA 17-00607S). For the actual analyses and experiments, the infrastructure of the SIX Center was used.

\section{REFERENCES}

[1] Fiala, P., Friedl, M., Hobst, L., Komarkova, T. (2014). Method of evaluating distribution, density and orientation of ferromagnetic electrically conducting fibers within composite material and detection device for making the same. Czech Patent Application PV2014-742.

[2] Wang, W., Dai, Y., Zhang, C., Gao, X., Zhao, M. (2016). Micromechanical modeling of fiber-reinforced composites with statistically equivalent random fiber distribution. Materials, 9 (8), 1-14.

[3] Giasin, K., Ayvar-Soberanis, S. (2016). Evaluation of workpiece temperature during drilling of GLARE fiber metal laminates using infrared techniques: Effect of cutting parameters, fiber orientation and spray mist application. Materials, 9 (8), 1-17.

[4] Zou, S., Wan, Z., Lu, L., Tang, Y. (2016). Experimental study on tensile properties of a novel porous metal fiber/powder sintered composite sheet. Materials, 9 (9), $1-11$.

[5] Mizukami, K., Mizutani, Y., Kimura, K., Sato, A., Todoroki, A., Suzuki, Y. (2016). Detection of in-plane fiber waviness in cross-ply CFRP laminates using layer selectable eddy current method. Composites Part A: Applied Science and Manufacturing, 82, 108-118.

[6] Zheng, K., Chang, Y.S., Wang, K.H., Yao, Y. (2016). Thermographic clustering analysis for defect detection in CFRP structures. Polymer Testing, 49, 73-81.

[7] Santoro, S., Drioli, E., Figoli, A. (2016). Development of novel ECTFE coated PP composite hollow-fiber membranes. Coatings, 6 (3), 1-12.

[8] Faifer, M., Ottoboni, R., Toscani, S., Ferrara, L. (2011). Nondestructive testing of steel-fiber-reinforced concrete using a magnetic approach. IEEE Transactions on Instrumentation and Measurement, 60 (5), 17091717.

[9] Chady, T., Enokizono, M., Sikora, R., Takeuchi, K., Kinoshita, T. (2002). Eddy current testing of concrete structures. International Journal of Applied Electromagnetics and Mechanics, 15, 33-37.

[10] Martinie, L., Roussel, N. (2011). Simple tools for fiber orientation prediction in industrial practice. Cement and Concrete Research, 41 (10), 993-1000.

[11] Shah, A.A., Ribakov, Y. (2011). Recent trends in steel fibered high-strength concrete. Materials and Design, 32 (8-9), 4122-4151.

[12] Ozyurt, N., Mason, T.O., Shah, S.P. (2006). Nondestructive monitoring of fiber orientation using AC-IS: An industrial-scale application. Cement and Concrete Research, 36 (9), 1653-1660.

[13] Szymanik, B., Frankowski, P.K., Chady, T., Azariah, C.R., Szczecin, J.C. (2015). Detection and inspection of steel bars in reinforced concrete structures using active infrared thermography with microwave excitation and eddy current. Sensors, 16 (2), 1-16.

[14] Hobst, L., Bílek, P., Vodička, J., Vala, J. (2014). Measurement of set fibre-concrete homogeneity in finished steel fibre-concrete structure of segmental tunnel lining. Advanced Materials Research, 1106, 4144. 
[15] Stratton, J.A. (1941). Electromagnetic Theory. McGraw-Hill.

[16] Fiala, P., Friedl, M., Hobst, L., Komarkova, T. (2016). A method and a detection device for evaluating the distribution, density and orientation of ferromagnetic, electrically conductive fibres in a composite material. Patent WO 2016070859 A1.

[17] Faifer, M., Ottoboni, R., Toscani, S., Ferrara, L. (2013). Low frequency electrical and magnetic methods for non-destructive analysis of fiber dispersion in fiber reinforced cementations composites. Sensors, 13 (1), 1300-1318.
[18] Komarkova, T. (2015). Design of methodology for nondestructive-testing of steel-reinforced-fiber- concrete. In Proceedings of the Conference on the Rehabilitation and Reconstruction of Buildings (CRRB 2015), vol. 714, 179-185.

[19] Komarkova, T., Friedl, M. (2015). Methodology for non-destructive evaluation of concentration and orientation of steel fibers in steel-fiber- reinforced concrete. In International Conference on Materials and Technology. Book of Abstracts, Ljublana, Slovenia, p. 136.

Received January 24, 2018 Accepted September 19, 2018 